\title{
MULTÍMETRO INTERFACEADO DE BAIXO CUSTO PARA AQUISIÇÃO DE DADOS
}

\author{
Eduardo M. Richter, Fábio R. P. Rocha e Lúcio Angnes*
}

Departamento de Química Fundamental, Instituto de Química, Universidade de São Paulo, Av. Prof. Lineu Prestes, 748, 05508-900 São Paulo - SP

Recebido em 14/3/03; aceito em 11/8/03

\begin{abstract}
A LOW COST MULTIMETER FOR DATA ACQUISITION. A digital multimeter ( U 240.00 on the national market) connected to a microcomputer by a RS-232 serial interface is proposed for data acquisition in equipment with analog output. Data are measured at the rate of 2 points per second and stored in text files by the software that accompanies the device, running in a Windows environment. The performance of the multimeter was verified by monitoring the transient signals generated in flow injection systems associated with fluorimetric, spectrophotometric and flame photometric detection. In addition, the performance of the proposed device was similar to that attained by employing an interface card with a 12-bit analog-to-digital converter for acquisition of the signals generated by a capillary electrophoresis equipment with oscillometric detection.
\end{abstract}

Keywords: data acquisition; digital multimeter; flow analysis.

\section{INTRODUÇÃO}

O interfaceamento a microcomputadores é uma tendência dos equipamentos analíticos disponíveis no mercado. Os microcomputadores são empregados para controle, aquisição e processamento dos dados, permitindo a obtenção de maior quantidade de informações, com elevada confiabilidade. Entretanto, equipamentos nos quais os dados são disponibilizados em leitores de cristal líquido e/ou em saídas analógicas são ainda usualmente encontrados em laboratórios de ensino e pesquisa. Em experimentos que resultam em grande quantidade de dados, o emprego desses equipamentos torna-se limitado e o seu desempenho certamente pode ser melhorado com o interfaceamento a microcomputadores.

Em comparação ao emprego de registradores potenciométricos, o interfaceamento apresenta como vantagens o custo relativamente menor e a possibilidade de se efetuar o processamento adicional dos dados em planilhas e editores gráficos. Para o interfaceamento, têm sido empregadas interfaces construídas em laboratório ${ }^{1,2}$, que não são acessíveis a todos os usuários, pois requerem conhecimento de eletrônica para sua construção. Por outro lado, as interfaces disponíveis comercialmente $^{3-5}$ apresentam custo relativamente alto e seu emprego requer algum conhecimento de programação de microcomputadores.

Neste trabalho, é proposto o emprego de um multímetro digital interfaceado a um computador como alternativa de baixo custo para a aquisição de dados em equipamentos com saída analógica. $\mathrm{O}$ dispositivo, que é facilmente adquirível no mercado nacional, tem custo similar ao das interfaces mais simples, porém com a importante vantagem de ser acompanhado por software para aquisição de dados e armazenamento em arquivos tipo texto.

\section{PARTE EXPERIMENTAL}

\section{Reagentes e soluções}

As soluções foram preparadas utilizando água deionizada em sistema de purificação NANOpure, Barnstead $\left(\mathrm{R} \geq 18 \mathrm{M} \Omega \mathrm{cm}^{-1}\right)$ e reagentes com grau analítico. Soluções estoque $10 \mathrm{mg} \mathrm{L}^{-1}$ quinino,

*e-mail: luangnes@iq.usp.br
$250 \mathrm{mg} \mathrm{L}^{-1} \mathrm{MnO}_{4}^{-}$e $100 \mathrm{mg} \mathrm{L}^{-1} \mathrm{Na}^{+}$foram preparadas a partir de sulfato de quinino, permanganato de potássio e cloreto de sódio. Solução $0,05 \mathrm{~mol} \mathrm{~L}^{-1} \mathrm{H}_{2} \mathrm{SO}_{4}$ foi empregada como transportador nas medidas por fluorescência. Solução $20 \mathrm{mmol} \mathrm{L}^{-1}$ ácido 2-N-[morfolino] etano sulfônico (MES), $20 \mathrm{mmol} \mathrm{L}^{-1}$ histidina (HIS) e $0,2 \mathrm{mmol} \mathrm{L}^{-1} \mathrm{de}$ brometo de N-cetil N-N-N trimetilamônio (CTAB), pH 6,2 foi empregada nas medidas por eletroforese capilar. Soluções de referência contendo 200, 400, 500, 600, 700 e $800 \mu \mathrm{g} \mathrm{L}^{-1}$ de quinino; 10, 20, 30, 40 e $50 \mathrm{mg} \mathrm{L}^{-1}$ de permanganato e $2,4,6,8$ e $10 \mathrm{mg} \mathrm{L}^{-1}$ de sódio foram preparadas por diluição das soluções estoque.

\section{Equipamentos e assessórios}

Os sistemas de análise por injeção em fluxo foram construídos empregando um injetor-comutador com barra deslizante e tubos de polietileno de $0,9 \mathrm{~mm}$ d.i. Um mini-compressor de ar do tipo bomba de diafragma, originalmente destinado ao borbulhamento de ar em aquários domésticos ${ }^{6}$, foi empregado para propulsão das soluções. Para a medida dos sinais, foram empregados fluorímetro (Turner, modelo 450), espectrofotômetro (Micronal, modelo B 342II) e fotômetro de chama (Digimed, modelo NK - 2004). Para as medidas por eletroforese capilar, foi utilizado um equipamento com detecção condutométrica sem contato (oscilométrica) construído em nosso laboratório ${ }^{7,8}$.

A aquisição de dados foi realizada com o multímetro modelo HC 608 (Hung Chung-Seoul, Korea), interfaceado a um microcomputador. Esse multímetro possui uma interface serial RS-232 e permite a aquisição de dados em intervalos de $0,5 \mathrm{~s}$. O multímetro é acompanhado de software para ambiente windows que gera arquivos de dados do tipo texto (máximo de 10.000 pontos), compatíveis com a maioria dos programas gráficos empregados para tratamento de dados.

\section{Procedimento}

As medidas em fluxo foram efetuadas em sistemas em linha única. Nos experimentos por fluorescência, foram empregadas soluções de quinino, o qual é excitado em $360 \mathrm{~nm}$ e apresenta fluorescência na região de $430 \mathrm{~nm}$. A solução transportadora foi ácido sulfúrico $0,05 \mathrm{~mol} \mathrm{~L}^{-1}\left(3,2 \mathrm{~mL} \mathrm{~min}^{-1}\right)$ e o volume injetado foi $100 \mu \mathrm{L}$. As 
medidas espectrofotométricas foram efetuadas em $525 \mathrm{~nm}$, empregando soluções de permanganato (volume injetado de $100 \mu \mathrm{L}$ ). Água foi empregada como transportador neste experimento e a vazão foi de $3 \mathrm{~mL} \mathrm{~min}^{-1}$. Nas medidas por fotometria de chama, foram empregadas soluções de cloreto de sódio, com volume injetado de $350 \mu \mathrm{L}$ e água à vazão 6,0 de $\mathrm{mL}$ minn $^{-1}$ como transportador.

Nas medidas por eletroforese capilar foi empregado um capilar de sílica fundida $(50 \mathrm{~cm})$ com diâmetro interno de $75 \mu \mathrm{m}$; voltagem de separação de - $15 \mathrm{kV}$, injeção por gravidade $(100 \mathrm{~mm}, 30 \mathrm{~s}) \mathrm{e}$ detecção por condutividade sem contato $(540 \mathrm{kHz})$. Uma solução de álcool combustível foi utilizada como amostra.

\section{RESULTADOS E DISCUSSÃO}

O multímetro foi conectado ao microcomputador através de uma interface serial RS-232 e à saída analógica dos equipamentos, permitindo a aquisição de dados em intervalos de $0,5 \mathrm{~s}$. Para a avaliação do desempenho, foram realizados três experimentos em sistemas de análise em fluxo com diferentes detectores. Na Figura 1 são apresentados os registros dos sinais obtidos em medidas por fluorescência (a), espectrofotometria (b) e fotometria de chama (c). Verifica-se que o multímetro apresentou desempenho adequado para a aquisição dos sinais transientes gerados em sistemas de análises em fluxo, sendo os sinais obtidos caracterizados por elevadas repetibilidade e freqüências de amostragem inerentes a estes sistemas.

Para demonstrar o desempenho do multímetro em relação a outro sistema de aquisição, comparou-se o sinal obtido em um sistema de eletroforese capilar com detecção condutométrica sem contato ${ }^{7,8}$, no qual a aquisição de dados foi realizada simultaneamente com uma interface comercial (Advanced PCL - 711B) e com o multímetro digital. A Figura 2 apresenta os sinais medidos simultaneamente com os diferentes dispositivos. Neste experimento, uma alíquota de álcool combustível foi utilizada como amostra, sendo identificados os picos de cloreto, nitrato, sulfato, acetato e lactato (padrão interno). Pode ser observado ainda, um outro pico de um analito desconhecido. Apesar de apresentar uma taxa de conversão consideravelmente menor que a interface PCL-711B, o desempenho do multímetro foi também bastante adequado para a aquisição do eletroferograma. $\mathrm{Na}$ prática, a taxa de conversão de $0,5 \mathrm{~s}$ é adequada para a aquisição de dados na maioria dos equipamentos analíticos.
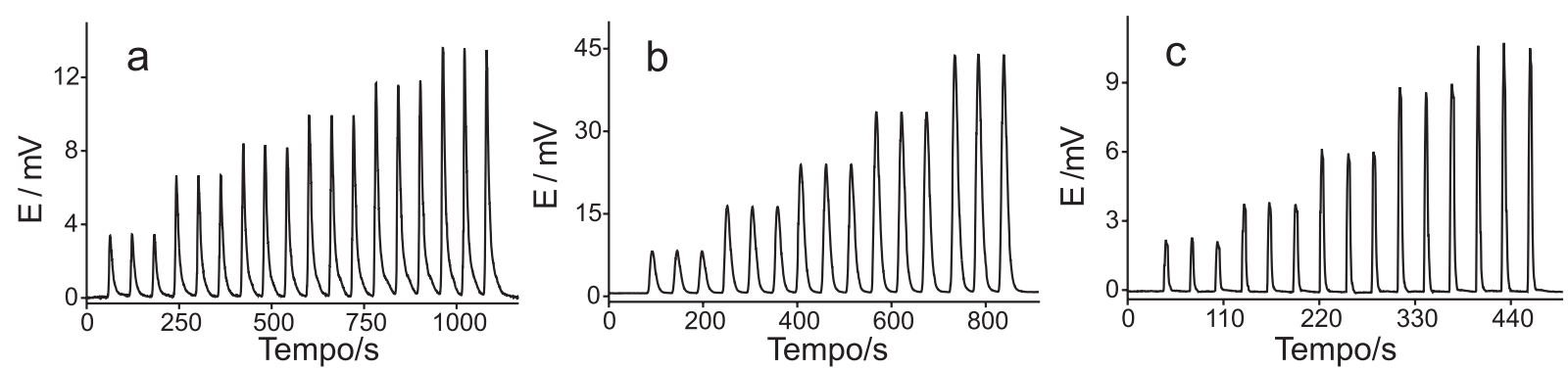

Figura 1. Sinais transientes obtidos em sistemas de análises por injeção em fluxo usando o multímetro para aquisição de dados: (a) medidas por fluorescência: soluções de quinino 200, 400, 500, 600, 700 e $800 \mu \mathrm{g} \mathrm{L}$; volume injetado - $100 \mu \mathrm{L}$; solução transportadora - 0,05 $\mathrm{mol} \mathrm{L}^{-1} \mathrm{em} \mathrm{H}_{2} \mathrm{SO}_{4}\left(3,2 \mathrm{~mL} \mathrm{~min}^{-1}\right)$; (b) medidas espectrofotométricas: soluções de permanganato 10,20, 30, 40 e $50 \mathrm{mg} \mathrm{L}^{-1}$; volume injetado - $100 \mu \mathrm{L}$; transportador $-\mathrm{H}_{2} \mathrm{O}(3 \mathrm{~mL} /$ min); (c) medidas por fotometria de chama: soluções de sódio 2, 4, 6, 8 e $10 \mathrm{mg} \mathrm{L}^{-1}$; volume injetado - $350 \mu \mathrm{L}$; transportador $-\mathrm{H}_{2} \mathrm{O}(6 \mathrm{~mL} / \mathrm{min})$
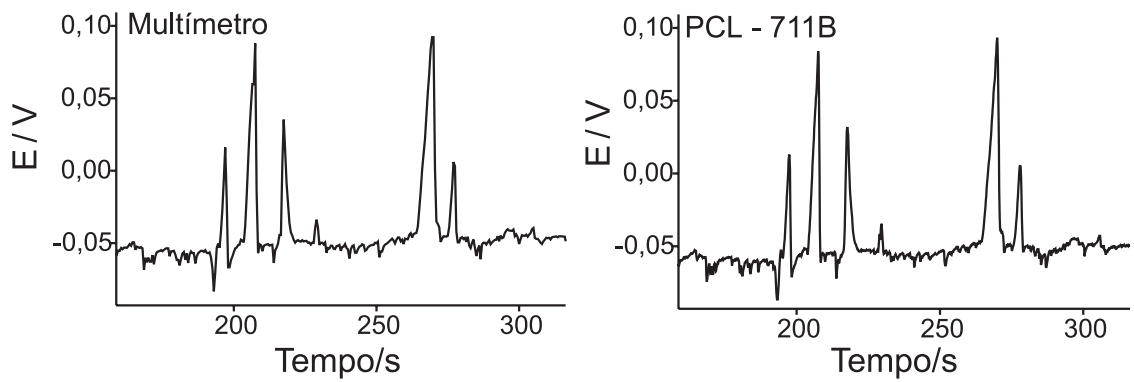

Figura 2. Aquisição simultânea de um eletroferograma empregando o multímetro e uma interface comercial (PCL-711B). Tampão de corrida - solução de MES/HIS (pH 6,2) 20 mmol L L e 0,2 mmol L-1 de CTAB, capilar de sílica fundida (50 cm) com diâmetro interno de $75 \mu \mathrm{m}$, voltagem de separação (- $15 \mathrm{kV})$, injeção por gravidade (100 mm, $30 \mathrm{~s})$, detecção por condutividade sem contato $(540 \mathrm{kHz})$

\section{CONCLUSÕES}

O multímetro digital apresentou um desempenho bastante satisfatório para a medida dos sinais transientes gerados em sistemas de análise em fluxo e eletroforese. O custo relativamente baixo ( U\$ 240.00), a eliminação da necessidade de elaborar um programa dedicado e a disponibilidade no mercado nacional fazem com que seja uma alternativa bastante atraente para a aquisição de dados em laboratórios de ensino, pesquisa e análise de rotina.

\section{AGRADECIMENTOS}

Os autores agradecem à FAPESP, CAPES e ao CNPq (projeto RENAMI) pelas bolsas e auxílios concedidos.

\section{REFERÊNCIAS}

1. Gutz, I. G. R.; Tese de Livre Docência, Universidade de São Paulo, Brasil, 1985.

2. Bellato, C. R.; Rohwedder, J. J. R.; Raimundo, I. M.; Pasquini, C.; J. Autom. Chem. 1996, 18, 7.

3. Korn, M.; Paim, A. P. S.; Barros, V. A. F., Reis, B. F.; Quim. Nova 1996, 19, 302 .

4. Rohwedder, J. J. R.; Pasquini, C.; Analyst 1998, 123, 1641.

5. Matos, R. C.; Angnes, L.; do Lago, C. L.; Instrum. Sci. Technol. 1998, 26, 451 .

6. Matos, R. C.; Gutz, I. G. R.; Angnes, L.; Fontenele, R. S.; Pedrotti, J. J.; Quim. Nova 2001, 24, 795.

7. da Silva, J. A. F.; do Lago, C. L.; Anal. Chem. 1998, 70, 4339.

8. da Silva, J. A. F.; do Lago, C. L.; J. Chromatogr., A 2002, 942, 249. 